Revised version [No. JMES-16-1381]

\title{
An Analytical Model for Predicting Permanent Plastic Deflection and Strain Distributions in Aluminium-alloy Plates Under Low Velocity Impact Loading
}

J B Bai ${ }^{1}$, J J Xiong ${ }^{1, *}$, R A Shenoi², M Liu ${ }^{1}$

1 School of Transportation Science and Engineering, Beihang University, Beijing, 100191, People’s Republic of China (*, corresponding author: jjxiong@buaa.edu.cn)

2 Southampton Marine and Maritime Institute, University of Southampton, Southampton, UK

\begin{abstract}
This paper proposes a new analytical model to predict plastic deformation and strain distributions in aluminium-alloy plates under low velocity impact loadings. The low velocity impact load on the fully clamped circular plate was idealized as a quasi-static normal point force acting at the center of plate. Based on apt geometrical approximation and assumptions, governing equations were established to predict the out-of-plane deflection and the radial tensile, radial and circumferential flexure strains in fully clamped conditions. From the deformation theory of plasticity, a new formula was derived to estimate the impact load by incorporating strain-energy approach, bilinear strain-hardening constitutive model and the one-dimensional Tresca yield criterion. Low velocity impact tests were performed to confirm the proposed model and good correlation was achieved between the predictions and actual experiments, demonstrating the practical and effective use of the proposed model.
\end{abstract}

Key words: yield; constitutive relation; aluminium-alloy plate; impact; strain energy

\section{NOMENCLATURE}

$A_{i} \quad$ transformation variable

$C_{i} \quad$ undetermined parameter.

D $\quad$ flexure stiffness

$E_{e} \quad$ elastic slope (or modulus) of material

$E_{p} \quad$ plastic slope of material 
impact height

$K_{r}$

radial curvature

$K_{\theta} \quad$ circumferential curvature

$m$ impact mass

$P$ impact load, or quasi-static normal point force acting at the center of circular plate

$Q_{r}$ shear force on the concentric circular cross-section of separated segment with a radius

of $r$

$r$

radial coordinate

$r_{0} \quad$ radius of circular plate

$S$

relative deviation of predictions from experiments

$t$

thickness of plate

$U$

total strain energy

$U_{t} \quad$ strain energy for radial tensile deformation

$U_{f} \quad$ strain energy for radial and circumferential flexures

w out-of-plane deflection of plate

$\bar{X}_{i} \quad$ nominal value of the general input variable

$\bar{Y} \quad$ nominal value of the response parameter

Z

vertical coordinate

$\theta \quad$ azimuth coordinate

$\delta \quad$ impact dent depth

$v \quad$ Poisson ratio

$\sigma_{r} \quad$ radial principal stress

$\sigma_{s} \quad$ yield strength of material

$\sigma_{z} \quad$ out-of-plane principal stress

$\sigma_{\theta} \quad$ circumferential principal stress 


$\begin{array}{ll}\varepsilon_{e} & \text { maximum elastic strain of material } \\ \varepsilon_{r} & \text { radial flexure strain } \\ \varepsilon_{t} & \text { radial tensile strain } \\ \varepsilon_{\theta} & \text { circumferential flexure strain } \\ \Pi_{0} & \text { impact energy, or } \Pi_{0}=m g H \\ \text { AA } & \text { aluminium alloy } \\ N S C_{X_{i}} & \text { normalized sensitivity coefficient for the input variable } X_{i}\end{array}$

\section{INTRODUCTION}

Structures made of thin metallic plates can be subjected to low velocity impact loading from events such as dropped maintenance tools. Such impacts could show up as dents in the thin metallic plates signifying plastic deformations. Such dents can have an effect on the static strength and fatigue behavior of the plated structures. Hereby, there is growing interest in investigating permanent plastic deformation problems for thin metallic plates under low impact velocity loading.

A significant body of literature is available dealing with the impact characteristics and permanent plastic deformation mechanism of thin metallic plate. These researches are classified and listed in Table 1 according to detailed issues. Impact experiments were conducted to reveal the effects of the factors of projectile shapes (e.g., blunt, hemispherical and conical noses ${ }^{[1,2]}$, thickness ${ }^{[2]}$ and aspect ratio $^{[3]}$ of metallic plates, and low temperature $e^{[4,5]}$ on the impact characteristics of metallic plates. Several analytical approaches/models have been proposed to predict the response and permanent plastic deflection of the metallic plate under low velocity impact loading by means of strain energy approach and non-linear strain-hardening behaviour depicted by the exponential Cowper-Symonds function $^{[6-9,15,18-21]}$, or the Johnson-Cook model ${ }^{[10]}$. FE analysis ${ }^{[11-13]}$ is also important for understanding the impact characteristics and permanent plastic deformation mechanism of metallic plates.

From the review, it is interesting to note that a number of parameters in the analytical models need to be determined from detailed experimental data or analysis. In addition, in order to produce the FE models, straight inclined segments or continuous mathematical functions have been used to depict 
more details such as the idealized projectile nose geometry and to represent the impact path and dent shape of metallic plate. The one drawback of the above FE models is their intensity and complexity. It stands to reason that it is desirable to have a technique to address the paucity of data in assessing impact characteristics and permanent plastic deformation mechanism of metallic plates for design purposes. In other words, it is desirable to craft a technique by which the permanent plastic deformation or impact dent depth of metallic plates under low velocity impact loading could expediently be predicted using only some basic material properties and geometrical dimensions, without any extensive experimental investigation. The purpose of this paper, therefore, is to develop a new analytical method for evaluating impact characteristics and permanent plastic deformation of thin AA (aluminium-alloy) plates only using basic material properties and geometrical dimensions.

\section{ANALYTICAL MODEL FOR PREDICTING IMPACT DEFORMATION}

In order to analyze the low velocity impact behaviours, the fundamental assumptions made in this paper are as follows:

(1) As is well known, the further strain arising from the travelling waves from the impact site out towards the plate periphery is negligibly small at low impact velocity as compared with that at high impact velocity. Consequently, the travelling wave effect is neglected at low impact velocity, which is up to about $20 \mathrm{~m} / \mathrm{s}$.

(2) Previous literature [10] shows that unlike the highly strain rate sensitive materials (e.g., mild steel, etc.), aluminium-alloys (AAs) are not sensitive to the strain rate effect. Low velocity impact does not induce a strain rate effect for the slightly sensitive material to strain rate. For this reason, the strain rate effect is ignored in low velocity impact analysis for aluminium-alloy (AA) plates. In such a case, low velocity impact load on a fully clamped circular AA plate is idealized as the quasi-static normal point force acting at the center of circular plate (shown in Figures 1 and 2a).

(3) Due to the symmetry of a fully clamped circular plate subjected to low velocity impact loading, the radial displacement and the rebound deformation are negligibly small in contrast to the out-of-plane deflection. Thus, the radial displacement and the rebound deformation are ignored, and the impact responses are approximated to only the radial tensile, and radial and circumferential flexure deformations. In reality, a number of analytical or empirical models ${ }^{[3,9,10,14,15,18-21]}$ also neglected the radial displacement in order to simplify these models; despite this, the predicted 
results using the aforementioned models correlated well with experiments. Thus, the same simplification is adopted in this work to craft a simplified method for preliminary design purposes.

(4) According to the Kirchhoff-love assumption, the normal stress $\sigma_{z}$ is ignored too.

From Figures 1 and 2a and Assumptions (2) and (3), it is obvious that for a circular AA plate subjected to a quasi-static normal point force of $P$ at the center of circular plate, the out-of-plane deflection $w$ is a function only for radial coordinate $r$. In order to analyze the out-of-plane deflection of the fully clamped circular plate, only a separated concentric circular segment with a radius of $r$ (shown in Figure 2b) is considered. From the force equilibrium of the separated concentric circular segment in the direction of $Z$ axis, one has

$$
2 \pi r Q_{r}=P
$$

where $r$ is the radial coordinate in cylindrical coordinate system. $Q_{r}$ is the shear force on the concentric circular cross-section of separated segment with a radius of $r . P$ is the quasi-static normal point force at the center of the circular plate.

In light of the definition of shear force $Q_{r}$ in linear elastic theory, it can be shown that

$$
Q_{r}=-D \frac{d}{d r}\left(\frac{d^{2} w}{d r^{2}}+\frac{1}{r} \frac{d w}{d r}\right)
$$

with

$$
D=\frac{E_{e} t^{3}}{12\left(1-v^{2}\right)}
$$

where $w, t$ and $D$ are respectively the out-of-plane deflection, thickness and flexure stiffness of plate. $E_{e}$ and $v$ are respectively the elastic slope and Poisson ratio of material.

Substituting Equation (2) into Equation (1) leads to

$$
-D \frac{d}{d r}\left(\frac{d^{2} w}{d r^{2}}+\frac{1}{r} \frac{d w}{d r}\right)=\frac{P}{2 \pi r}
$$

Solving Equation (4), it is possible to have

$$
w=\frac{P}{8 \pi D}\left(r^{2} \ln r+C_{1} r^{2}+C_{2} \ln r+C_{3}\right)
$$

where $C_{1}, C_{2}$ and $C_{3}$ are the undetermined parameters.

Equation (5) is the out-of-plane deflection function of fully clamped circular plate subjected to a 
quasi-static normal point force at the center of circular plate. The parameters $C_{1}, C_{2}$ and $C_{3}$ in Equation (5) can be determined using the boundary conditions as follows in a two-stages process.

(a) From the symmetry principle (or Assumption (3)), the maximum out-of-plane deflection appears at the center of circular plate, i.e.,

$$
\left.\frac{d w}{d r}\right|_{r=0}=0
$$

(b) On the basis of the fully clamped boundary condition, it can be shown that

$$
\left\{\begin{array}{l}
\left.w\right|_{r=r_{0}}=0 \\
\left.\frac{d w}{d r}\right|_{r=r_{0}}=0
\end{array}\right.
$$

where $r_{0}$ is the radius of circular plate.

Substituting Equation (5) into Equations (6) and (7) results in

$$
\left\{\begin{array}{l}
C_{1}=-\frac{1}{2}-\ln r_{0} \\
C_{2}=0 \\
C_{3}=\frac{1}{2} r_{0}^{2}
\end{array}\right.
$$

Substituting Equation (8) into Equation (5) induces

$$
w=\frac{P}{8 \pi D}\left(r^{2} \ln \frac{r}{r_{0}}+\frac{r_{0}^{2}-r^{2}}{2}\right)
$$

Equation (9) shows the out-of-plane deflection function on the fully clamped circular plate subjected to low velocity impact loading, with unknown $P$. Like the other mathematical models (e.g., the zero-order Bessel function of the first kind ${ }^{[15]}$, etc.) for depicting the deflection profile of a circular plate, Equations (1) to (9) seek to craft the deformation profile of the impacted plate, more rationally and more easily. From Equation (9), it can be shown that the impact dent depth of the circular plate is

$$
\delta=\left.w\right|_{r=0}=\frac{r_{0}^{2} P}{16 \pi D}
$$

Obviously, the result calculated from Equation (10) is the maximum value of impact dent depth, neglecting the elastic unloading. From Equations (9) and (10), it is apparent that the out-of-plane deflection $w$ and impact dent depth $\delta$ on a fully clamped circular plate are with respect to the 
unknown quasi-static normal point force (i.e., impact load) of $P$. The unknown impact load of $P$ necessitates the solving from the deformation theory of plasticity by incorporating strain-energy approach, elastic-plastic strain-hardening constitutive model and yield criterion. If the impact load $P$ is solved and determined, then the out-of-plane deflection and impact dent depth of circular plate are obtained by using Equations (9) and (10).

Based on the definition the radial tensile strain $\varepsilon_{t}$, it is possible to have

$$
\varepsilon_{t}=\frac{\sqrt{d w^{2}+d r^{2}}-d r}{d r}=\frac{1}{2}\left(\frac{d w}{d r}\right)^{2}
$$

where $\varepsilon_{t}$ is the radial tensile strain.

Substituting Equation (9) into Equation (11), one has

$$
\varepsilon_{t}=\frac{P^{2}}{32 \pi^{2} D^{2}} r^{2}\left(\ln \frac{r}{r_{0}}\right)^{2}
$$

Letting

$$
\begin{gathered}
r_{1}=\frac{r}{r_{0}}\left(0 \leq r_{1}<1\right) \\
\mathrm{A}_{1}=\frac{r_{0}^{2}}{32 \pi^{2} D^{2}}
\end{gathered}
$$

then Equation (12) becomes

$$
\varepsilon_{t}=A_{1} P^{2} r_{1}^{2}\left(\ln r_{1}\right)^{2}
$$

Again, with aid of the definitions of radial and circumferential curvatures, it is feasible to have

$$
\left\{\begin{array}{c}
K_{r}=\frac{d^{2} w}{d r^{2}}=\frac{P}{4 \pi D}\left(\ln r_{1}+1\right) \\
K_{\theta}=\frac{1}{r}\left(\frac{d w}{d r}\right)=\frac{P}{4 \pi D} \ln r_{1}
\end{array}\right.
$$

where $K_{r}$ and $K_{\theta}$ are respectively the radial and circumferential curvatures.

From Equation (16), the radial and circumferential flexure strains can be then attained as

$$
\left\{\begin{array}{c}
\varepsilon_{r}=K_{r} z=\frac{P z}{4 \pi D}\left(\ln r_{1}+1\right) \\
\varepsilon_{\theta}=K_{\theta} z=\frac{P z}{4 \pi D} \ln r_{1}
\end{array}\right.
$$

where $\varepsilon_{r}$ and $\varepsilon_{\theta}$ are respectively the radial and circumferential strains. $Z$ is the vertical 
coordinate in cylindrical coordinate system.

\section{ANALYTICAL SOLUTION FOR PREDICTING IMPACT LOAD}

From the elastic-plastic theory, the plastic flow law of a material is generally depicted by incorporating the elastic-plastic strain-hardening constitutive model and the yield criterion. From an engineering viewpoint, the linearized elastic-plastic constitutive relation is argued to be apt and effective in depicting plastic flow law of material. Hence a bilinear elastic-plastic strain-hardening constitutive model with an elastic slope of $E_{e}$ and a maximum elastic strain $\varepsilon_{e}$ as well as a plastic slope of $E_{p}$ (shown in Figure 3) is implemented to describe the plastic flow law of material in this work. Moreover, it is proven that the Von Mises criterion seems more appropriate and effective for isolating the yield condition in contrast to the Tresca rule ${ }^{[23]}$. The one drawback of the Von Mises criterion is the intensity and complexity in identifying the yield condition owing to its nonlinear form. In fact, the Tresca rule, which although simplistic and linear, is effective in isolating the yield condition. Thus, the Tresca rule is used for identifying the yield condition in this work. In the cylindrical coordinate system, the Tresca criterion can be written as

$$
\left\{\begin{array}{l}
\left|\sigma_{r}-\sigma_{z}\right|=\sigma_{s} \\
\left|\sigma_{z}-\sigma_{\theta}\right|=\sigma_{s} \\
\left|\sigma_{\theta}-\sigma_{r}\right|=\sigma_{s}
\end{array}\right.
$$

where $\sigma_{r}, \sigma_{\theta}$ and $\sigma_{z}$ are respectively the principal stresses in the radial, circumferential and vertical directions. $\sigma_{s}$ is the yield strength of material.

According to the Kirchhoff-Love assumption, it can be shown that

$$
\sigma_{z}=0
$$

Substituting Equation (19) into Equation (18) deduces

$$
\left\{\begin{array}{c}
\left|\sigma_{r}\right|=\sigma_{s} \\
\left|\sigma_{\theta}\right|=\sigma_{s} \\
\left|\sigma_{\theta}-\sigma_{r}\right|=\sigma_{s}
\end{array}\right.
$$

Equation (20) is the one-dimensional Tresca yield criterion (i.e. the maximum principal stress yield criterion). From Equation (20), it is expedient and effective to identify the yield condition of plate by comparing the principal stresses of $\sigma_{r}$ and $\sigma_{\theta}$ with the yield strength of $\sigma_{s}$. 
Based on the plastic flow law of material depicted by incorporating the bilinear elastic-plastic strain-hardening constitutive model and the one-dimensional Tresca yield criterion (or Equation(20)), it can be shown that the strain energy for radial tensile deformation within the plate is $^{[23]}$

$$
U_{t}=\int_{V}\left(\int \sigma_{t} d \varepsilon_{t}\right) d V=\int_{0}^{r_{0}} \int_{-\frac{t}{2}}^{\frac{t}{2}} \int_{0}^{2 \pi}\left[\frac{E_{e} \varepsilon_{e}^{2}}{2}+\sigma_{s}\left(\varepsilon_{t}-\varepsilon_{e}\right)+\frac{E_{p}\left(\varepsilon_{t}-\varepsilon_{e}\right)^{2}}{2}\right] r d \theta d z d r
$$

where $E_{p}$ and $\varepsilon_{e}$ are respectively the plastic slope and maximum elastic strain of material in the bilinear elastic-plastic strain-hardening model.

Substituting Equation (15) into Equation (21) induces

$$
U_{t}=A_{2} P^{4}+A_{3} P^{2}+A_{4}
$$

with

$$
\begin{gathered}
A_{2}=\frac{\pi E_{p} t r_{0}^{2} A_{1}^{2}}{324} \\
A_{3}=\frac{\pi t r_{0}^{2} A_{1}\left(\sigma_{s}-E_{p} \varepsilon_{e}\right)}{16} \\
A_{4}=\pi t \varepsilon_{e} r_{0}^{2}\left(\frac{E_{e}+E_{p}}{2} \cdot \varepsilon_{e}-\sigma_{s}\right)
\end{gathered}
$$

Similarly, the strain energy for radial and circumferential flexure can be shown to be

$$
\begin{aligned}
U_{f} & =\int_{0}^{r_{0}} \int_{-\frac{t}{2}}^{\frac{t}{2}} \int_{0}^{2 \pi}\left[\frac{E_{e} \varepsilon_{e}^{2}}{2}+\sigma_{s}\left(\varepsilon_{r}-\varepsilon_{e}\right)+\frac{E_{p}\left(\varepsilon_{r}-\varepsilon_{e}\right)^{2}}{2}\right] r d \theta d \theta d z \\
& +\int_{0}^{r_{0}} \int_{-\frac{t}{2}}^{\frac{t}{2}} \int_{0}^{2 \pi}\left[\frac{E_{e} \varepsilon_{e}^{2}}{2}+\sigma_{s}\left(\varepsilon_{\theta}-\varepsilon_{e}\right)+\frac{E_{p}\left(\varepsilon_{\theta}-\varepsilon_{e}\right)^{2}}{2}\right] r d \theta d \theta d z
\end{aligned}
$$

Substituting Equation (17) into Equation (26) deduces

$$
U_{f}=A_{5} P^{2}+A_{6} P+A_{7}
$$

with

$$
\begin{gathered}
A_{5}=\frac{E_{p} r_{0}^{2} t^{3}}{768 \pi D^{2}} \\
A_{6}=\frac{r_{0}^{2} t^{2}}{8 D}\left(\sigma_{s}-E_{p} \varepsilon_{e}\right)\left(\frac{1}{2}+\frac{1}{e^{2}}\right)
\end{gathered}
$$




$$
A_{7}=\pi t \varepsilon_{e} r_{0}^{2}\left(E_{e} \varepsilon_{e}+E_{p} \varepsilon_{e}-2 \sigma_{s}\right)
$$

From Equations (22) and (27), it is possible to have total strain energy as

$$
U=U_{t}+U_{f}=A_{2} P^{4}+\left(A_{3}+A_{5}\right) P^{2}+A_{6} P+\left(A_{4}+A_{7}\right)
$$

For a free drop-hammer, the impact energy accounting for the effect of impact dent depth can be expressed as

$$
\Pi=m g(H+\delta)
$$

where $m$ is the impact mass. $g$ is the gravity acceleration. $H$ is the impact height.

In general, the energy dissipations resulted from the air resistance, friction and thermal effects are negligibly small in contrast to the strain energy. As a result, from an engineering viewpoint, all impact energy is assumed to be changed into the strain energy and absorbed in the plate through radial tensile, radial and circumferential flexure deformations.

$$
U=\Pi
$$

or

$$
A_{2} P^{4}+\left(A_{3}+A_{5}\right) P^{2}+\left(A_{6}+A_{8}\right) P+A_{4}+A_{7}+A_{9}=0
$$

with

$$
A_{8}=-\frac{m g r_{0}^{2}}{16 \pi D}
$$

and

$$
A_{9}=-m g H=-\Pi_{0}
$$

where $\Pi_{0}$ is the impact energy, or $\Pi_{0}=m g H$.

The value of impact load $P$ can be obtained by solving Equation (34). As mentioned previously, if $P$ is solved and determined, the out-of-plane deflection $w$ and the impact dent depth $\delta$ can then be determined from Equations (9) and (10). Actually, in the case of dynamic loads, the inertia of the plate itself and the striking mass change with time. Even though presumably the final state of a plate would be the same as the predictions of a quasi-static analysis by using the same total energy input and deformation profile and by ignoring any potential history effects, they would not be the same for the sensitive material to the strain rate. Thus, as mentioned in assumption (2), the analytical solution in this work is suited only for the slightly sensitive materials to the strain rate 
effect.

\section{COMPARISONS BETWEEN EXPERIMENTS AND PREDICTIONS}

\subsection{Verifying example 1}

As shown in previous literature [10], low velocity impact tests were conducted on AA 5083-H116 circular plates with a radius of $125 \mathrm{~mm}$ and three different thicknesses of $3 \mathrm{~mm}, 5 \mathrm{~mm}$ and $6 \mathrm{~mm}$, by using a gas gun projectile launching system with an impact mass of $5.0 \mathrm{~kg}$. The mechanical properties and specifications as well as experimental data of AA 5083-H116 circular plates are shown in Tables 2 and 3. From mechanical properties and plate specifications listed in Table 2 and 3, the impact dent depths and loads are predicted by using Equations (10) and (34) respectively (shown in Table 3). From Table 3, it is seen that the predicted values of impact dents using the proposed method are higher than the experimental values. This is because the influence of the strain component $d u / d r$ arising from the radial displacement $u$ and the high-order terms of Taylor

expansion for $\left(\sqrt{d w^{2}+d r^{2}}-d r\right) / d r$ on the radial tensile strain $\varepsilon_{t}$ are neglected. This causes the values of radial tensile strain in the model to be lower than the actual ones. Thus, the plate in the model needs more deformation to absorb the same impact energy.

\subsection{Verifying example 2}

Low velocity impact tests were carried out on fully clamped AA 2524-T3 circular plates at room temperature and moisture by using a free drop-hammer impact device (shown in Figure 4). The geometry and dimensions of specimen are shown in Figure 5. The mechanical properties of material and specifications are shown in Table 4. As shown in Table 4, the radius and thickness of the fully clamped circular plate are $11.5 \mathrm{~mm}$ and $1.6 \mathrm{~mm}$, respectively. A conical nose was employed in low velocity impact tests. The mass of drop-hammer was $8.23 \mathrm{~kg}$. Meanwhile, the central circular hole with a radius of $11.5 \mathrm{~mm}$ was fabricated on the rigid fixture base and the support fixture. The specimens were symmetrically and fully clamped on the rigid fixture base through the support fixture and four steel bolts.

According to the ASTM D7136/D7136M-07 ${ }^{[22]}$, three sets of impact tests were conducted under three different levels of impact energy $\Pi_{0}$ and at least five specimens were employed for each set of impact tests. During tests, all specimens were struck by the free drop-hammer at a specific level 
of impact energy, which was controlled by adjusting the impact height of drop-hammer from the rigid fixture base. The dent depths on the impacted specimens were measured using a square caliper. The experimental results are shown in Figures 6 and Table 5.

Again, from Equations (10) and (34) together with mechanical properties of material and specifications listed in Table 4, the impact dent depths on fully clamped AA 2524-T3 circular plates are predicted (shown in Figure 6 and Table 5). From Figure 6 and Table 5, it is evident that the values derived from the proposed model are less stiff than those shown in the experimental ones.

\subsection{Strain analysis}

Substituting the mechanical properties of material and specifications (listed in Table 4) into Equations (15) and (17), the strain distributions in radial tensile, radial and circumferential directions are obtained for fully clamped AA 2524-T3 circular plate at low impact velocity (shown in Figures 7 to 9). From Figures 7 to 9, it can be seen that the maximum circumferential flexure strain decreases with the increasing relative radial distance of $r / r_{0}$. In contrast, the maximum radial flexure strain increases with the increasing relative radial distance of $r / r_{0}$. Unlike the maximum radial and circumferential flexure strains, the radial tensile strain firstly increases and then decreases with the increasing relative radial distance of $r / r_{0}$, and there exists an inflection point on the curve of radial tensile strain versus relative radial distance.

\subsection{Discussion}

Geometrical and material parameters of the specimen and impact energy affected the dent depth during tests. A sensitivity analysis is conducted to determine the effect of all input parameters on the dent depth using the new model proposed in this paper. Normalized sensitivity coefficient (NSC) is defined as ${ }^{[24]}$

$$
N S C_{X_{i}}=\left(\frac{\Delta Y}{\bar{Y}} \frac{\bar{X}_{i}}{\Delta X_{i}}\right)^{2}
$$

where $N S C_{X_{i}}$ is the normalized sensitivity coefficient of the input variable $X_{i}, \bar{X}_{i}$ is the nominal value of the general input variable, and $\bar{Y}$ is the nominal value of the response parameter. Since the sensitivity coefficients of all input variables (i.e. geometrical and material parameters of the specimen and impact energy) are normalized relative to the same nominal value of the response parameter (i.e. dent depth), it is convenient to estimate and compare the sensitivity effect of each variables. The verifying example 2 is used in the sensitivity analysis, and a small change of the 
input variable is assumed to be $5 \%$. The calculated normalized sensitivity coefficients are shown in Figure 10. From Figure 10, it is evident that the dent depth is most sensitive to the plate thickness, especially for the lower impact loading case (e.g. $\Pi_{0}=2.5 \mathrm{~J}$ ). The dent depth has almost the same sensitivity to the impact energy, plate thickness and yield strength. This indicates that even a small change of the plate thickness can lead to a relative significant influence on the dent depth. That could be the reason for large relative deviation for a few cases.

Jones $^{[14]}$ also proposed an analytical solution for the same problem considered in this paper. In the two verifying examples, the predicted impact dent depths using the Jones' model are also obtained (shown in Tables 3 and 5). From Tables 3 and 5, it is clear that the predicted dent depths using the new model proposed this paper are higher than experimental results. This can be attributed to the simplification of the radial tensile strain in the new model that is mentioned at the end of the section 4.1, especially for the case of the plate with thickness $1.6 \mathrm{~mm}$ and under the ultra lower impact loading (e.g. the case of $\Pi_{0}=2.5 \mathrm{~J}$ listed in Table 5). However, the new model seems to have a better prediction accuracy than the Jones' model for the cases of thicker plates. The new model and the Jones’ model seem to be complementary in predictions of the dent depth.

Babaei and Darvizeh ${ }^{[15]}$ also proposed an analytical model to predict the plastic deformation of clamped circular plates under impulsive loading. Two explosive conditions (i.e. uniform load and localized load) were considered in their model, and the Cowper-Symonds empirical equation with two material constants $D$ and $q$ was used in the model. In their model and experiments, the plates were made from the mild steel with various thickness and radii. Thus, the specific material constants $D$ and $q$ for mild steel should be chosen or determined to predict the plastic deformation. However, in this work, the plates were made from AA 2524-T3, and the material constants $D$ and $q$ for AA 2524-T3 are unavailable at present.

From the above comparisons, it is clear that through Equations (10) and (34), the impact dent depth and strain distributions on fully clamped circular AA plates subjected to low-velocity impact loading could expediently be predicted without any significant experimental investigation. In other words, only the input of basic material properties and specifications of fully clamped circular AA plate is needed to predict impact dent depth and strain distributions. A reasonable correlation is achieved between the predictions using the proposed model and actual experiments. 


\section{CONCLUSIONS}

The focus of this paper has been to develop a new analytical method to predict the permanent plastic deformation and strain distributions on fully clamped circular AA plate subjected to low-velocity impact loading, by incorporating strain energy approach, bilinear strain-hardening constitutive model and one-dimensional Tresca yield criterion. Low velocity impact tests were conducted to confirm the new model. The new model has been proven successfully and reasonable correlation is achieved between predictions and actual experiments. The new model is argued to be a valid and rational basis for evaluating the permanent plastic deformation and strain distributions in fully clamped circular AA plates at low impact velocity ranged up to $13.2 \mathrm{~m} / \mathrm{s}$.

\section{ACKNOWLEDGEMENTS}

This project was supported by the National Natural Science Foundation of China (Grant No. 51375033 and 51405006).

\section{REFERENCES}

[1] Børvik T, Hopperstad OS, Berstad T, Langseth M. Perforation of 12mm thick steel plates by 20mm diameter projectiles with flat, hemispherical and conical noses Part I: Experimental study. International Journal of Impact Engineering, 2002; 27: 19-35.

[2] Jones N, Paik JK. Impact perforation of aluminium alloy plates. International Journal of Impact Engineering, 2012; 48: 46-53.

[3] Jones N, Birch RS, Duan R. Low-Velocity Perforation of Mild Steel Rectangular Plates With Projectiles Having Different Shaped Impact Faces. Journal of Pressure Vessel Technology, 2008; 130: 1-8.

[4] Rodriguez-Martinez JA, Pesci R, Rusinek A, Arias A, Zaera R, Pedroche D.A. Thermo-mechanical behaviour of TRIP 1000 steel sheets subjected to low velocity perforation by conical projectiles at different temperatures. International Journal of Solids and Structures, 2010; 47: 1268-1284.

[5] Rodriguez-Martinez JA, Rusinek A, Arias A. Thermo-viscoplastic behavior of 2024-T3 aluminium sheets subjected to low velocity perforation at different temperatures. Thin-Walled 
Structures, 2011; 49: 819-832.

[6] Forrestal MJ, Brar NS, Luk V K. Penetration of strain-hardening targets with rigid Spherical-Nose rods. Journal of Applied Mechanics, 1991; 58:7-10.

[7] Zaera R, Arias A, Navarro C. Analytical modelling of metallic circular plates subjected to impulsive loads. International Journal of Solids and Structures, 2002; 39: 659-672.

[8] Chen LB, Yang JL. Analytical evaluation of permanent deflection of a thin circular plate struck normally at its center. Acta Mechanica Solida Sinica, 2007; 20: 117-122.

[9] Jones N. Impact loading of ductile rectangular plates. Thin-Walled Structures, 2012; 50: 68-75.

[10]Mohotti D, Ali M, Ngo T, Lu J, Mendis P, Ruan D. Out-of-plane impact resistance of aluminium plates subjected to low velocity impacts. Materials and Design, 2013; 50: 413-426.

[11]Borvik T, Hopperstad OS, berstad T, Langseth M. Numerical simulation of plugging failure in ballistic penetration. International Journal of Solids and Structures, 2001; 38: 6241- 6264.

[12]Dey S, Bovika T, Hopperstad OS, Langseth M. On the influence of constitutive relation in projectile impact of steel plates. International Journal of Impact Engineering, 2007; 34: 464-486.

[13]Iqbal MA, Gupta G, Gupta NK. 3D numerical simulations of ductile targets subjected to oblique impact by sharp nosed projectiles. International Journal of Solids and Structures, 2010; 47: 224-237.

[14]Jones N. Dynamic inelastic response of strain rate sensitive ductile plates due to large impact, dynamic pressure and explosive loadings. International Journal of Impact Engineering, 2014; 74: 3-15.

[15]Babaei H, Darvizeh A. Analytical study of plastic deformation of clamped circular plates subjected to impulsive loading. Journal of Mechanics of Materials and Structures, 2012; 7(4): $309-22$.

[16]Cloete TJ, Nurick GN. On the influence of radial displacements and bending strains on the large deflections of impulsively loaded circular plates. International Journal of Mechanical Sciences, 2014; 82: 140-148.

[17]Babaei H, Mostofi TM, Alitavoli M. Experimental and analytical investigation into large ductile transverse deformation of monolithic and multi-layered metallic square targets struck normally by rigid spherical projectile. Thin-Walled Structures, 2016; 107: 257-265.

[18]Babaei H, Mostofi TM. New dimensionless numbers for deformation of circular mild steel 
plates with large strains as a result of localized and uniform impulsive loading. Proceedings of the Institution of Mechanical Engineers Part L: Journal of Materials: Design and Applications, 2016; 0(0): 1-15.

[19]Babaei H, Mostofi T M, Armoudli E. On dimensionless numbers for the dynamic plastic response of quadrangular mild steel plates subjected to localized and uniform impulsive loading. Proceedings of the Institution of Mechanical Engineers Part E: Journal of Process Mechanical Engineering. 2016; 0(0)1-12.

[20]Mostofi TM, Babaei H, Alitavoli M. Theoretical analysis on the effect of uniform and localized impulsive loading on the dynamic plastic behaviour of fully clamped thin quadrangular plates. Thin-Walled Structures, 2016; 109: 367-376.

[21]Mostofi TM, Babaei H, Alitavoli M, Hosseinzadeh S. On dimensionless numbers for predicting large ductile transverse deformation of monolithic and multi-layered metallic square targets struck normally by rigid spherical projectile. Thin-Walled Structures, 2017; 112: 118-24.

[22]ASTM D7136/D7136M-07. Standard test method for measuring the damage resistance of a fiber-reinforced polymer matrix composite to a drop-weight impact event. American Society for Testing and Materials, 2007

[23] Xu BY. Concise course of plastic theory. Beijing: Tsinghua University Press; 1981.

[24]Qureshi BA, Zubair SM. A comprehensive design and rating study of evaporative coolers and condensers. Part II. Sensitivity analysis. International Journal of Refrigeration, 2006(4): 659-668.

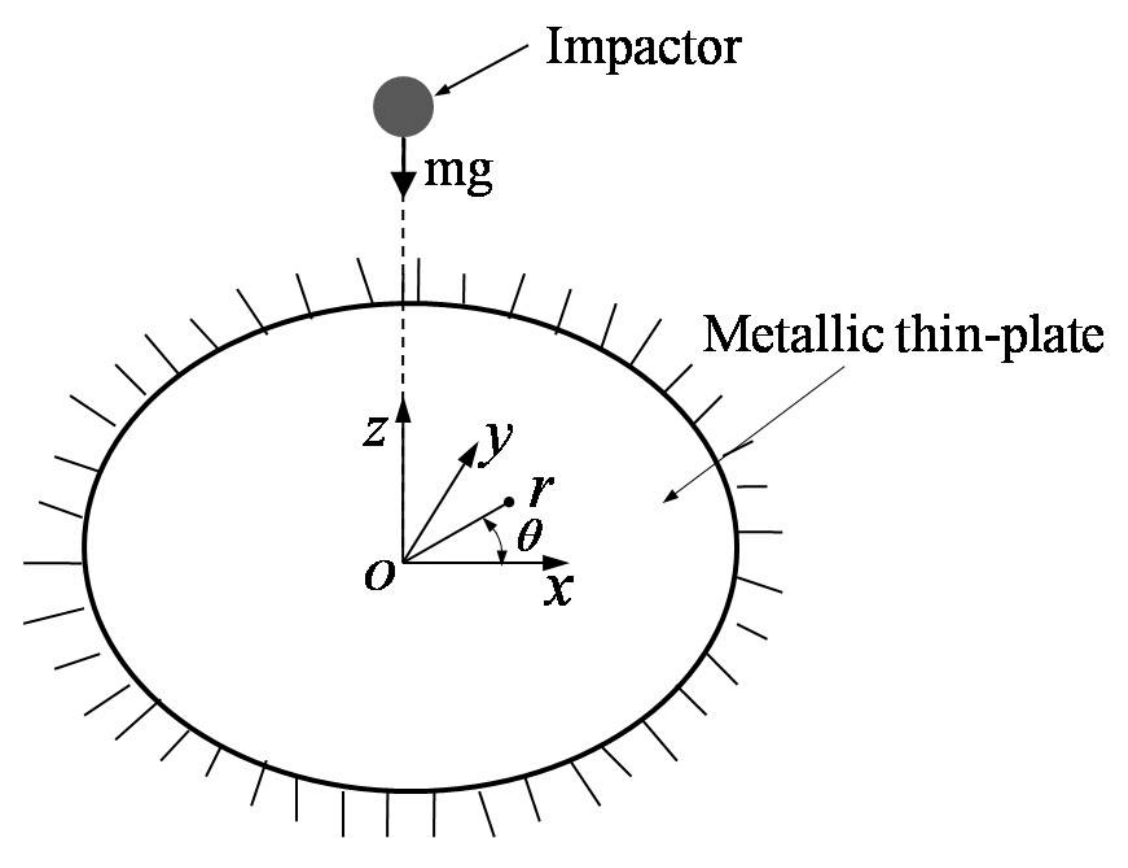


Figure 1 Fully clamped circular plate subjected to low velocity impact

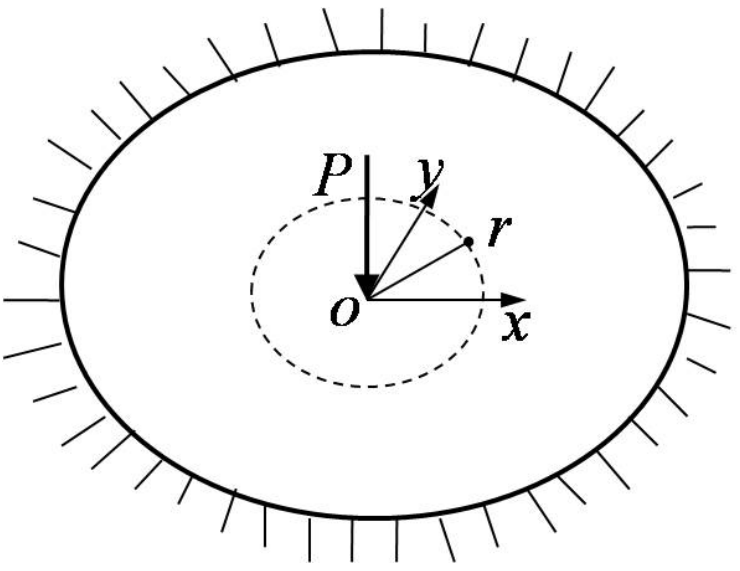

(a) Idealized fully clamped circular plate

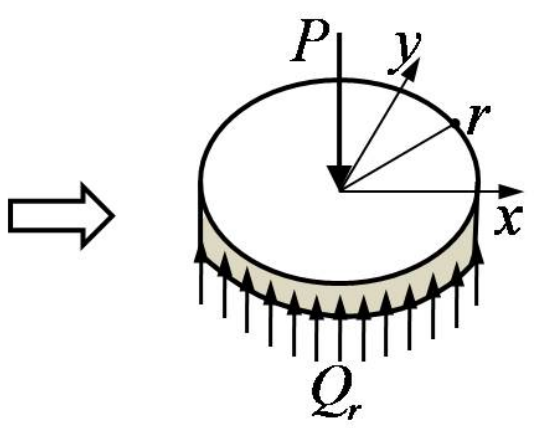

(b) Separated concentric circular segment

Figure 2 Idealized geometrical model

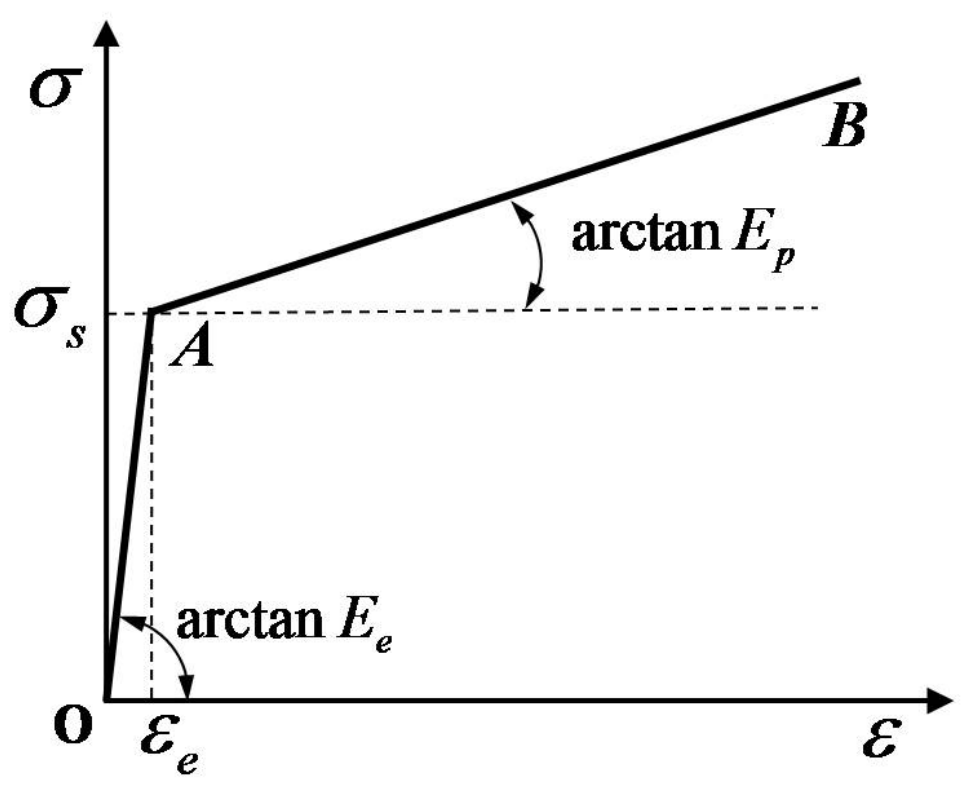

Figure 3 Bilinear elastic-plastic strain-hardening constitutive model 

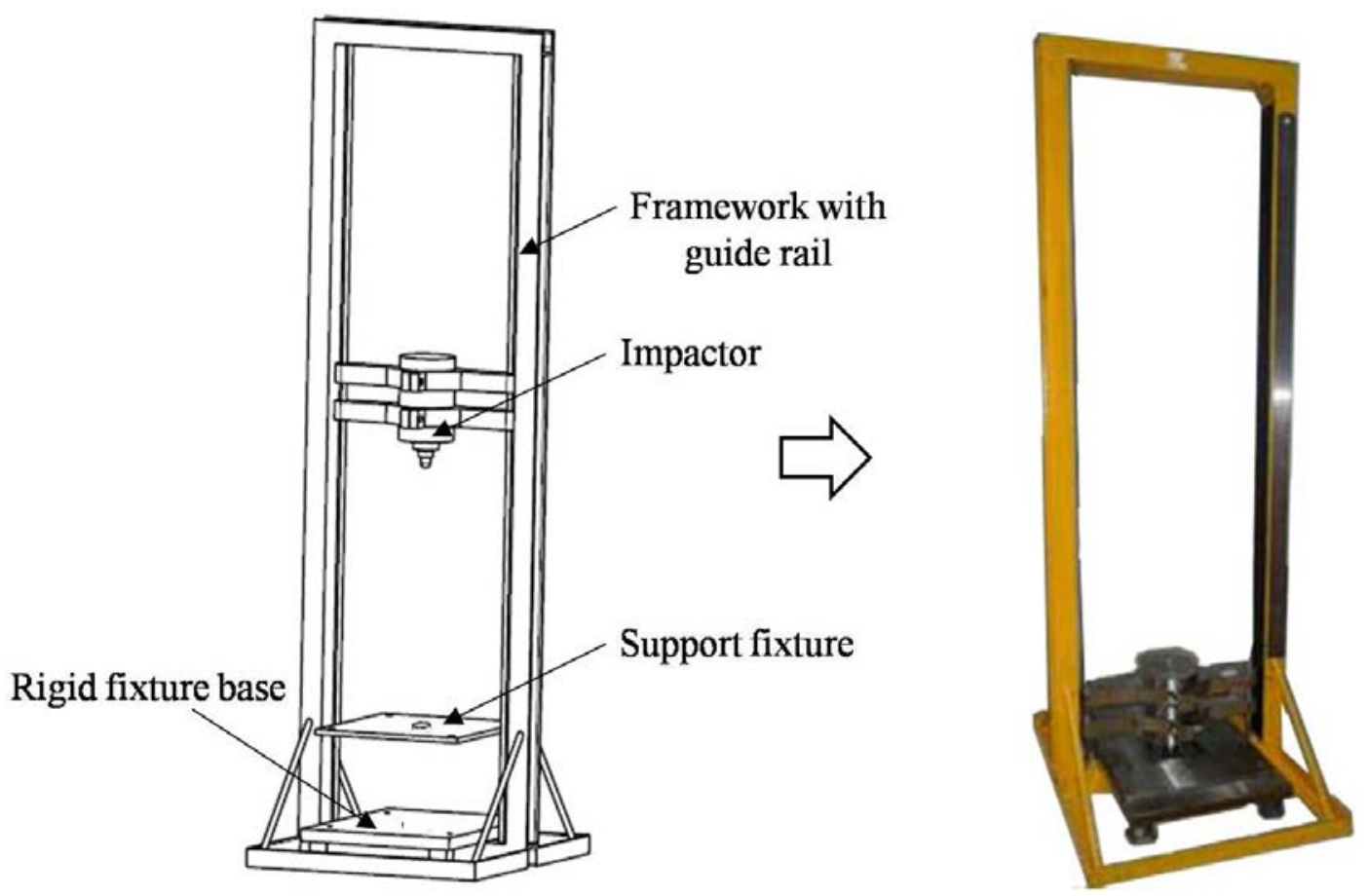

Figure 4 Free drop-hammer impact device
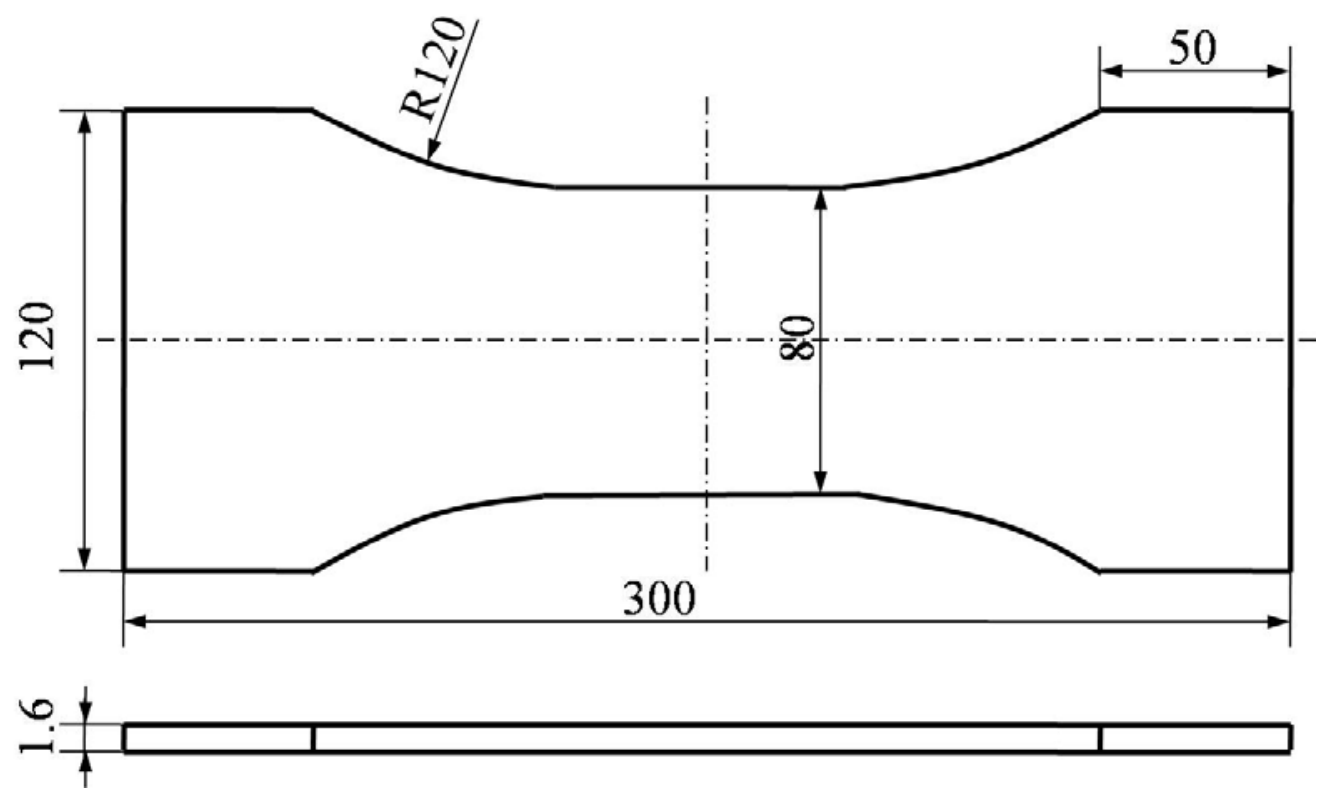

Figure 5 Geometry and dimensions for specimen (unit: $\mathrm{mm}$ ) 


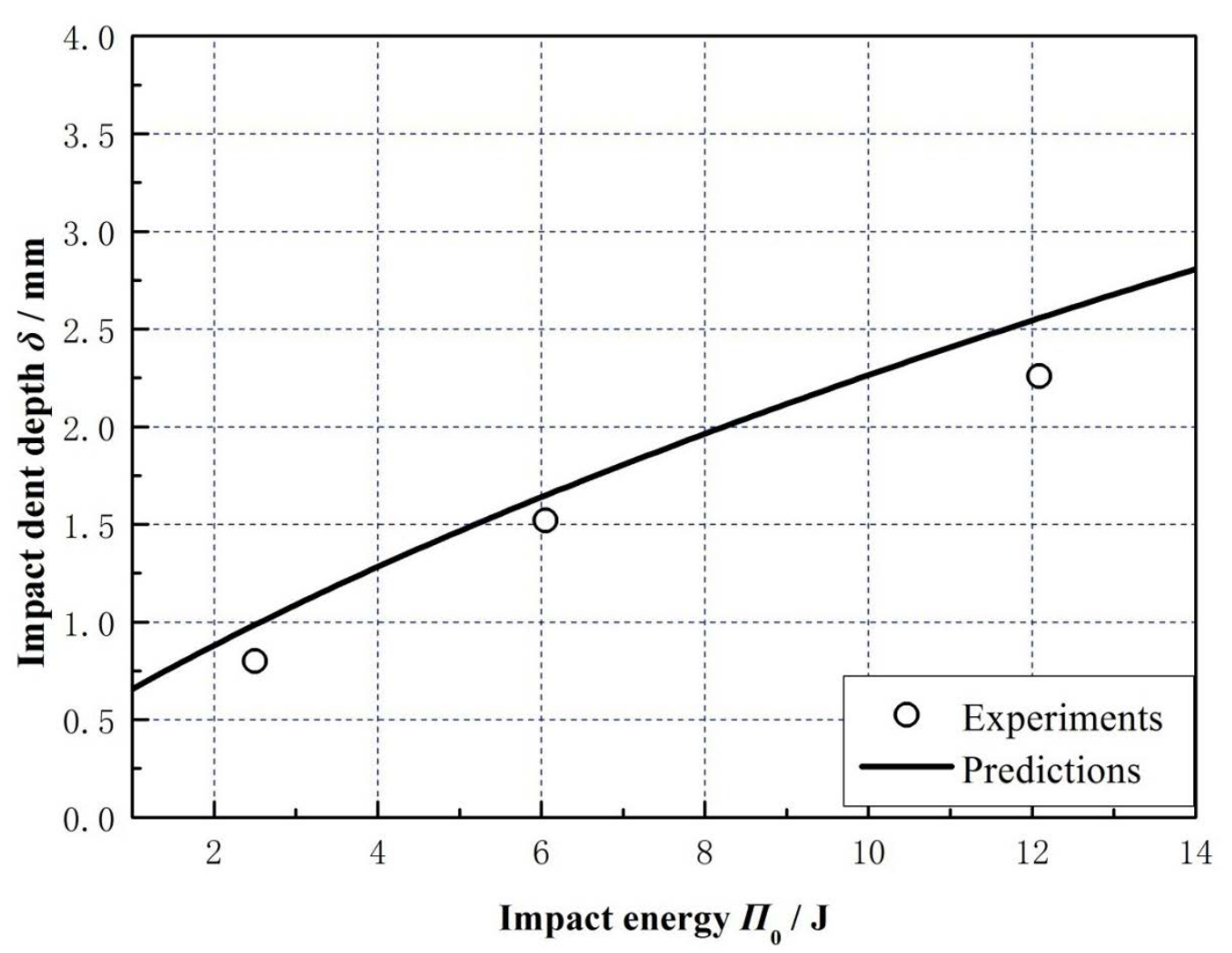

Figure 6 Impact dent depth versus impact energy

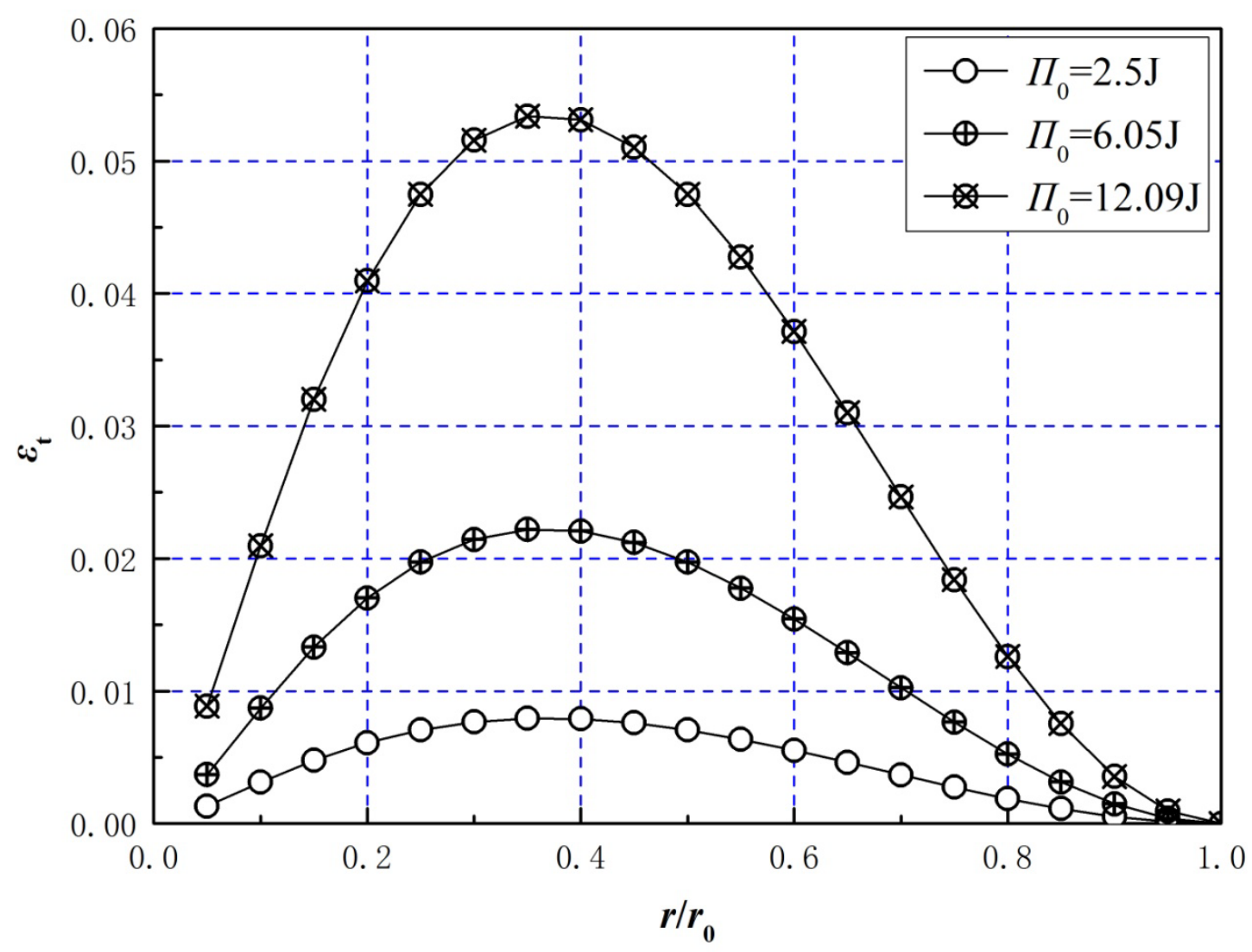

Figure 7 Radial tensile strain versus relative radial distance 


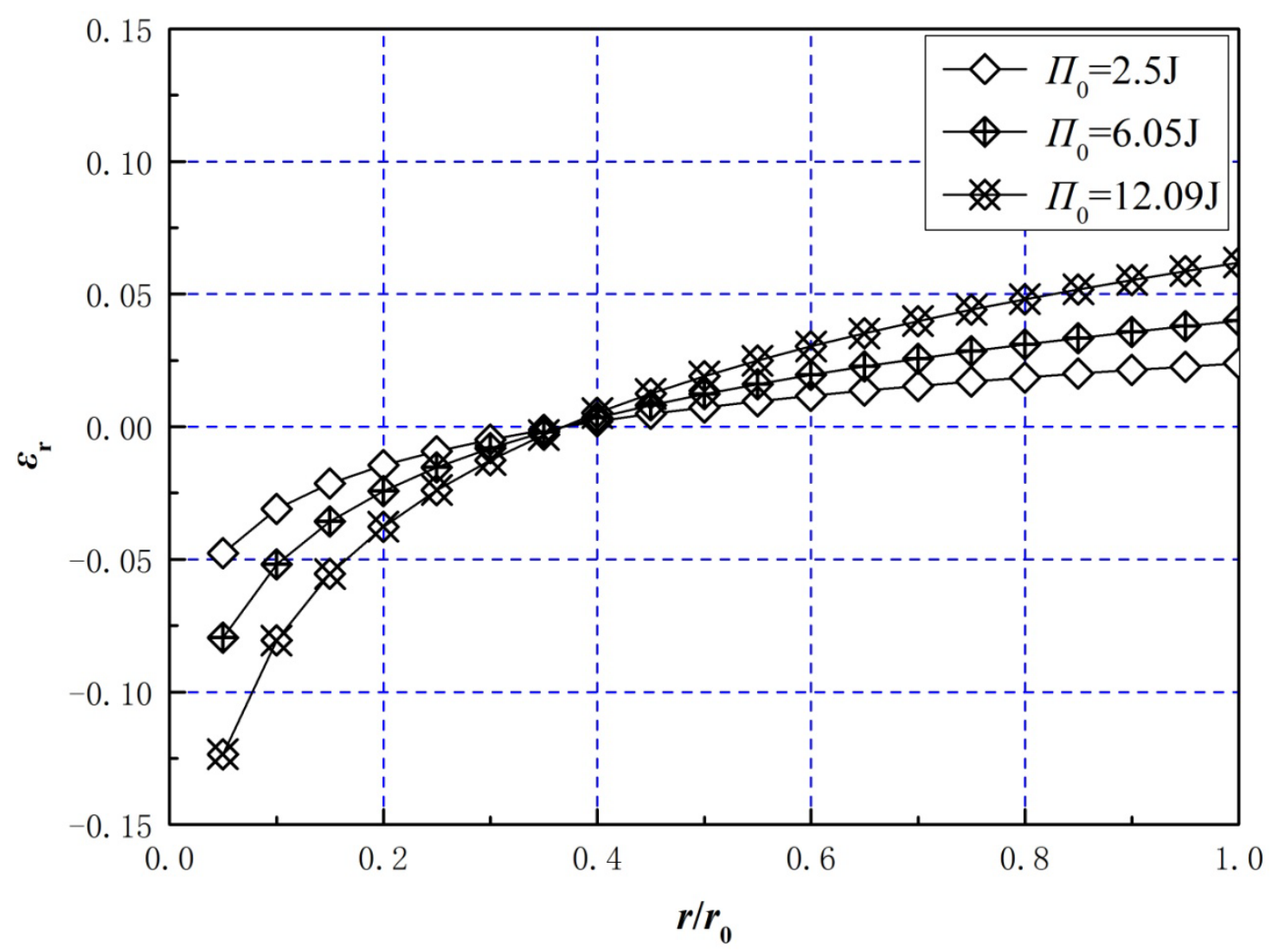

Figure 8 Maximum radial flexure strain versus relative radial distance

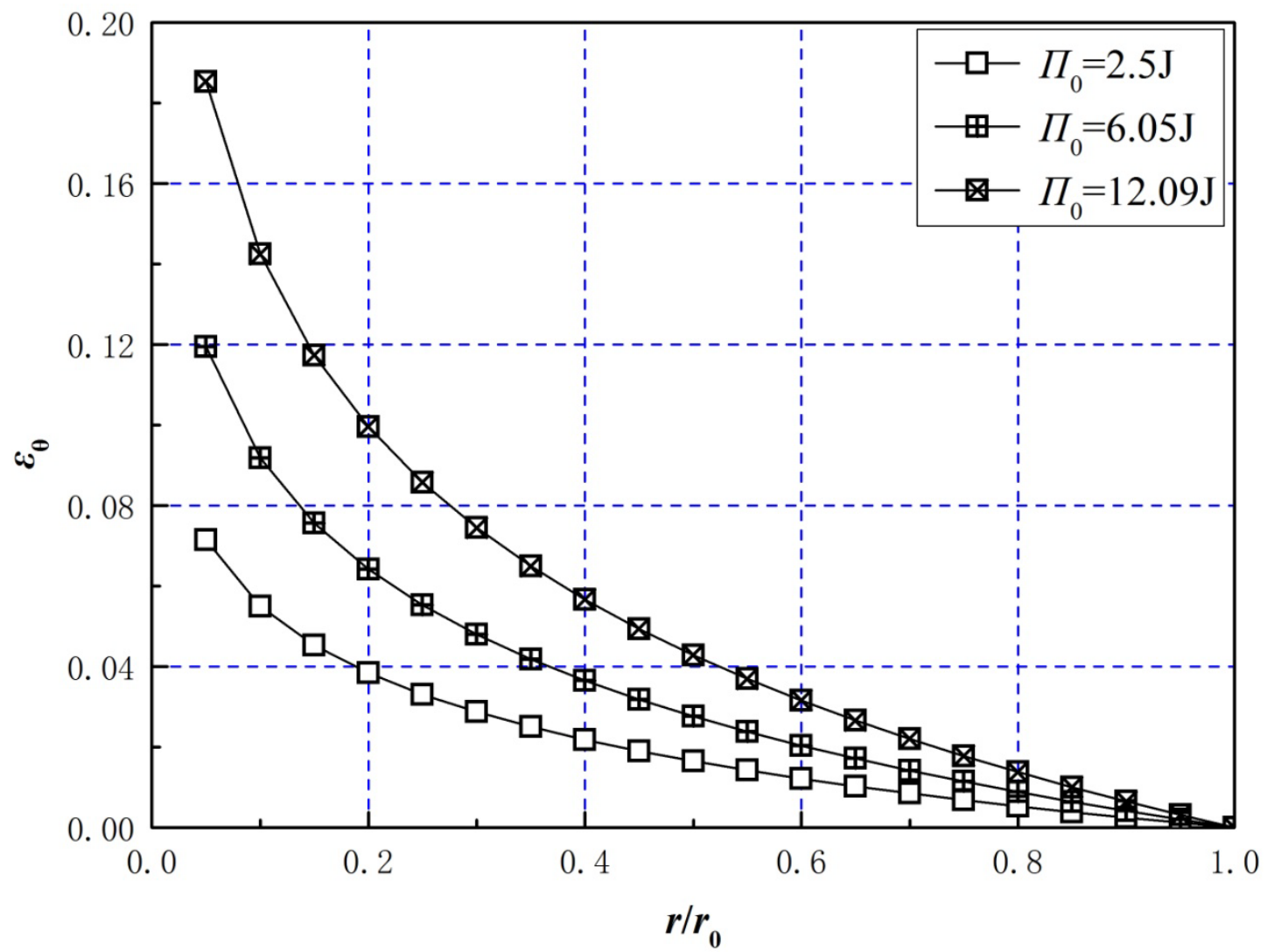

Figure 9 Maximum circumferential flexure strain versus relative radial distance 


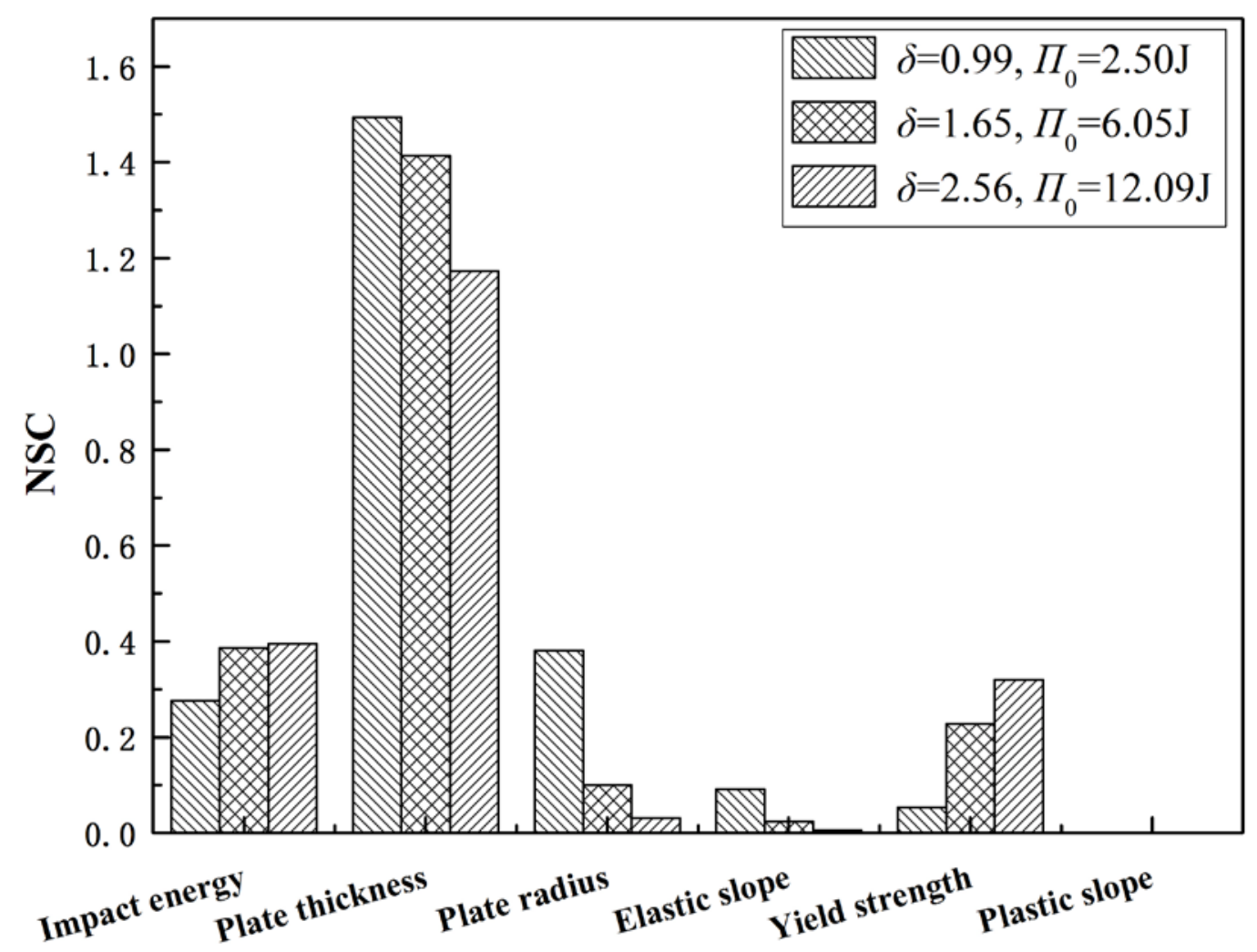

Figure 10 Normalized sensitivity coefficient (NSC) 
Table 1 Impact analysis on plates using experimental, analytical and FE methods

\begin{tabular}{|c|c|c|c|c|}
\hline \multicolumn{2}{|c|}{ Issues } & Experimental work & Analytical or & FE models \\
\hline \multirow{3}{*}{ Material } & Steel & $\begin{array}{c}\text { [1] [3] [4] [6] [11] } \\
{[12][15][17][18]} \\
{[20]}\end{array}$ & $\begin{array}{c}\text { [6] [7] [8] [9] [13] } \\
\text { [14] [15] [17] } \\
{[18][19]}\end{array}$ & [11] [12] [13] \\
\hline & Aluminium alloy & [2] [5] [6] [10] [17] & $\begin{array}{c}\text { [5] [6] [7] [10] } \\
\text { [13] [17] }\end{array}$ & [10] [13] \\
\hline & $\begin{array}{l}\text { Multi-layered } \\
\text { metallic }\end{array}$ & [17] [21] & [17] [21] & \\
\hline \multirow[b]{2}{*}{ Configuration } & $\begin{array}{l}\text { Projectile shapes } \\
\text { and dimensions }\end{array}$ & $\begin{array}{c}{[1][3][4][5][6]} \\
{[11][12]}\end{array}$ & $\begin{array}{c}{[5][6][9][13]} \\
{[14]}\end{array}$ & [11] [12] [13] \\
\hline & $\begin{array}{c}\text { Plate geometry and } \\
\text { dimensions }\end{array}$ & $\begin{array}{c}\text { [2] [3] [6] [10] [15] } \\
{[16][17][20][21]}\end{array}$ & $\begin{array}{c}\text { [6] [7] [8] [9] [14] } \\
\text { [15] [16] [17] } \\
\text { [18] [19] [20] } \\
\text { [21] }\end{array}$ & [10] \\
\hline \multirow[t]{2}{*}{ Velocity } & Low velocity & $\begin{array}{c}{[2][3][4][5][10]} \\
{[15][21]}\end{array}$ & $\begin{array}{c}{[5][7][8][9][10]} \\
{[14][15][21]}\end{array}$ & [10] \\
\hline & High velocity & [1] [6] [12] [17] & [6] [13] [17] & [11] [12] [13] \\
\hline \multirow[t]{2}{*}{$\begin{array}{l}\text { Boundary } \\
\text { condition }\end{array}$} & Fully clamped & $\begin{array}{c}\text { [1] [2] [3] [4] [5] } \\
\text { [6] [10] [11] [12] } \\
\text { [15] [17] [20] [21] }\end{array}$ & $\begin{array}{c}{[5][6][7][8][9]} \\
{[10][13][14]} \\
{[15][17][18]} \\
{[19][20][21]}\end{array}$ & $\begin{array}{c}{[10][11][12]} \\
{[13]}\end{array}$ \\
\hline & Simply supported & - & [9] [14] & - \\
\hline \multirow[t]{2}{*}{ Failure mode } & Plastic dent & $\begin{array}{c}{[4][10][15][16]} \\
{[17][20][21]}\end{array}$ & $\begin{array}{c}\text { [7] [8] [9] [10] } \\
{[15][16][17]} \\
{[18][19][20]} \\
{[21]}\end{array}$ & [10] \\
\hline & Perforation & $\begin{array}{c}{[1][2][3][4][5]} \\
{[6][11][12]}\end{array}$ & [5] [6] [13] & [11] [12] [13] \\
\hline
\end{tabular}




\begin{tabular}{|c|c|c|c|c|}
\hline \multirow{2}{*}{ Temperature } & $\begin{array}{c}\text { High or low } \\
\text { temperatures }\end{array}$ & {$[4][5]$} & {$[5]$} & - \\
\hline
\end{tabular}

Table 2 Mechanical properties for AA5083-H116 ${ }^{[10]}$

\begin{tabular}{|c|c|c|c|c|}
\hline$\sigma_{s}(\mathrm{MPa})$ & $E_{e}(\mathrm{MPa})$ & $E_{p}(\mathrm{MPa})$ & $v$ & $\varepsilon_{e}$ \\
\hline 200 & 68400 & 375 & 0.3 & 0.0029 \\
\hline
\end{tabular}

Table 3 Specifications and impact dent depths

\begin{tabular}{|c|c|c|c|c|c|c|c|}
\hline $\begin{array}{l}\text { Radius } \\
r_{0}(\mathrm{~mm})\end{array}$ & $\begin{array}{c}\text { Thickness } \\
t \text { (mm) }\end{array}$ & $\begin{array}{l}\text { Impact } \\
\text { energy } \\
\Pi_{0}(\mathrm{~J})\end{array}$ & $\begin{array}{l}\text { Experiments }^{[10]} \\
\qquad \delta(\mathrm{mm})\end{array}$ & $\begin{array}{c}\text { New } \\
\text { model } \\
\delta(\mathrm{mm})\end{array}$ & $\begin{array}{l}\text { Relative } \\
\text { deviation }\end{array}$ & $\begin{array}{c}\text { Jones' } \\
\text { model }^{[14]} \\
\delta(\mathrm{mm})\end{array}$ & $\begin{array}{l}\text { Relative } \\
\text { deviation }\end{array}$ \\
\hline 125 & 3 & 203.4 & 12.80 & 15.31 & $19.6 \%$ & 11.71 & $-8.48 \%$ \\
\hline 125 & 3 & 193.6 & 12.75 & 15.04 & $18.0 \%$ & 11.37 & $-10.82 \%$ \\
\hline 125 & 3 & 208.4 & 12.95 & 15.45 & $19.3 \%$ & 11.89 & $-8.21 \%$ \\
\hline 125 & 5 & 378.8 & 13.20 & 14.08 & $6.7 \%$ & 10.8 & $-17.84 \%$ \\
\hline 125 & 5 & 400.1 & 13.05 & 14.41 & $10.4 \%$ & 11.24 & $-13.86 \%$ \\
\hline 125 & 5 & 402.0 & 13.12 & 14.44 & $10.0 \%$ & 11.28 & $-14.05 \%$ \\
\hline 125 & 6 & 436.9 & 12.08 & 13.03 & $7.9 \%$ & 9.83 & $-18.63 \%$ \\
\hline 125 & 6 & 429.0 & 12.70 & 12.92 & $1.7 \%$ & 9.71 & $-23.57 \%$ \\
\hline 125 & 6 & 435.6 & 12.75 & 13.01 & $2.0 \%$ & 9.81 & $-23.06 \%$ \\
\hline
\end{tabular}

Table 4 Mechanical properties and specifications for AA2524-T3

\begin{tabular}{|c|c|c|c|c|c|c|}
\hline$\sigma_{s}(\mathrm{MPa})$ & $E_{e}(\mathrm{MPa})$ & $E_{p}(\mathrm{MPa})$ & $v$ & $\varepsilon_{e}$ & $t(\mathrm{~mm})$ & $r_{0}(\mathrm{~mm})$ \\
\hline 324 & 71000 & 50 & 0.35 & 0.0045 & 1.6 & 11.5 \\
\hline
\end{tabular}


Table 5 Impact dent depths for AA2524-T3 plate

\begin{tabular}{|c|c|c|c|}
\hline Impact energy $\Pi_{0}(\mathrm{~J})$ & 2.50 & 6.05 & 12.09 \\
\hline \multirow{2}{*}{ Experiments $\delta(\mathrm{mm})$} & 0.79 & 1.55 & 2.31 \\
\cline { 2 - 4 } & 0.81 & 1.54 & 2.30 \\
\cline { 2 - 4 } & 0.80 & 1.55 & 2.20 \\
\cline { 2 - 4 } & 0.79 & 1.50 & 2.24 \\
\hline Mean $\delta(\mathrm{mm})$ & 0.80 & 1.47 & 2.24 \\
\hline New model $\delta(\mathrm{mm})$ & 0.99 & 1.52 & 2.26 \\
\hline Relative deviation & $23.80 \%$ & $8.60 \%$ & $13.30 \%$ \\
\hline Jones' model ${ }^{[14]} \delta(\mathrm{mm})$ & 0.77 & 1.56 & 2.57 \\
\hline Relative deviation & $-3.75 \%$ & $2.63 \%$ & $13.7 \%$ \\
\hline
\end{tabular}

